\title{
Adolescent binge ethanol treatment alters adult brain regional volumes, cortical extracellular matrix protein and behavioral flexibility
}

\author{
Leon Garland Coleman Jr, M.D., Ph.D. ${ }^{a}$, Wen Liu, MD, Ph.D. ${ }^{a}$, Ipek Oguz, Ph.D. ${ }^{a, c}$, Martin \\ Styner, Ph.D. ${ }^{b, c}$, and Fulton T. Crews, Ph.D. ${ }^{a, b}$ \\ Leon Garland Coleman: leon_coleman@med.unc.edu; Wen Liu: wen_liu@med.unc.edu; Ipek Oguz: ipek@cs.unc.edu; \\ Martin Styner: styner@cs.unc.edu; Fulton T. Crews: ftcrews@med.unc.edu \\ aBowles Center for Alcohol Studies, University of North Carolina at Chapel Hill CB\# 7178, Chapel \\ Hill, NC 27599-7178, United States \\ bepartment of Psychiatry, University of North Carolina at Chapel Hill, Chapel Hill, NC 27599, \\ United States \\ 'Department of Computer Science, University of North Carolina at Chapel Hill, Chapel Hill, NC \\ 27599, United States
}

\begin{abstract}
Adolescents binge drink more than any other age group, increasing risk of disrupting the development of the frontal cortex. We hypothesized that adolescent binge drinking would lead to persistent alterations in adulthood. In this study, we modeled adolescent weekend underage bingedrinking, using adolescent mice (post-natal days [P] 28-37). The adolescent intermittent binge ethanol (AIE) treatment includes 6 binge intragastric doses of ethanol in an intermittent pattern across adolescence. Assessments were conducted in adulthood following extended abstinence to determine if there were persistent changes in adults. Reversal learning, open field and other behavioral assessments as well as brain structure using magnetic imaging and immunohistochemistry were determined. We found AIE did not impact adult Barnes Maze learning. However, AIE did cause reversal learning deficits in adults. AIE also caused structural changes in the adult brain. AIE was associated with adulthood volume enlargements in specific brain regions without changes in total brain volume. Enlarged regions included the orbitofrontal cortex (OFC, 4\%), cerebellum (4.5\%), thalamus (2\%), internal capsule (10\%) and genu of the corpus callosum (7\%). The enlarged OFC volume in adults after AIE is consistent with previous imaging studies in human adolescents. AIE treatment was associated with significant increases in the expression of several extracellular matrix (ECM) proteins in the adult OFC including WFA $(55 \%)$, Brevican (32\%), Neurocan (105\%), Tenacin-C (25\%), and HABP (5\%). These findings are consistent with AIE causing persistent changes in brain structure that could contribute to a lack of behavioral flexibility.
\end{abstract}

(C) 2013 The Authors. Published by Elsevier Inc. All rights reserved.

Corresponding Author: Dr. Fulton T. Crews, John Andrews Distinguished Professor, Professor of Pharmacology and Psychiatry, Director, Bowles Center for Alcohol Studies CB\# 7178, University of North Carolina at Chapel Hill, School of Medicine, Chapel Hill, N.C. 27599-7178, 919-966-5678, fax: 919-966-5679, ftcrews@ med.unc.edu.

Publisher's Disclaimer: This is a PDF file of an unedited manuscript that has been accepted for publication. As a service to our customers we are providing this early version of the manuscript. The manuscript will undergo copyediting, typesetting, and review of the resulting proof before it is published in its final citable form. Please note that during the production process errors may be discovered which could affect the content, and all legal disclaimers that apply to the journal pertain. 


\section{Keywords}

underage drinking; MRI; extracellular matrix; alcohol; neurocan; behavioral flexibility

\subsection{Introduction}

Adolescent human drinkers participate in heavy and binge drinking ( $>5$ drinks/episode) at substantially higher rates than adults (Ehlers et al., 2011; Lucantonio et al., 2012; SAMSA, 2007), with the rate of binge-drinking peaking during adolescence (Ehlers et al., 2011). Alcohol consumption during adolescence is highly prevalent as $8 \%$ of $8^{\text {th }}$ grade, $16 \%$ of 10 th grade, and $25 \%$ of $12^{\text {th }}$ grade adolescent individuals reported heavy episodic drinking (i.e., $<5$ consecutive alcohol drinks per episode) over the past 2 weeks (Johnston et al., 2009). This heavy drinking pattern persists into college as $44 \%$ of students report binge drinking every 2 weeks with $19 \%$ reporting more than 3 binge drinking episodes per week (O'Malley et al., 1998; Wechsler et al., 1995). Early onset of alcohol use (<13 years of age) is associated with increased drinking frequency and physical violence (Gruber et al., 1996). Further, large population studies find that across the teenage years the earlier the age of drinking onset the greater the risk of lifetime alcohol use disorder (Zhu et al., 2010) and lifetime alcohol related violence and injuries (Hingson and Winter, 2003; White et al., 2011). Adolescents with alcohol use disorders have deficits in executive functioning (Brown et al., 2000; Hanson et al., 2011; Tapert and Brown, 2000) consistent with frontal cortical dysfunction. Although studies have found that heavy drinking among adolescent males increases impulsivity the following year in those individuals predisposed to adolescenttypical impulsivity (White et al., 2011), human studies are all confounded by the difficulty in distinguishing a pre-existing condition that leads to alcohol abuse from an alcohol induced dysfunction that alters the individual beyond the period of intoxication.

Adolescence is a developmental period associated with maturation of cognitive ability, personality and frontal cortical executive functions (Crews et al., 2007; Gong et al., 2012; Spear, 2000). The frontal cortex continues to develop throughout adolescence and is uniquely sensitive to ethanol neurotoxicity (Crews et al., 2000). Adolescent rat ethanol treatment results in greater frontal cortical damage than adults as well as other alterations in adult brain neurotransmission (Basak and Bandyopadhyay, 2013; Coleman et al., 2011; Sharma and Bhatia, 2004; Torchilin, 2001), electrophysiological activity and sleep wave patterns (Escobar-Chavez et al., 2006), reduced forebrain cholinergic-choline acetyltransferase + neurons (Coleman et al., 2011; Ehlers et al., 2011) and reduced hippocampal neurogenesis (Ehlers et al., 2011). Further, adolescent rat ethanol treatment increases alcohol intake (Fabio et al., 2013; Pascual et al., 2007) and risk-taking preference (Qin et al., 2013), as well as impairing conditioned discrimination, object recognition (Pascual et al., 2007), and reversal learning (Coleman et al., 2011). These studies support the hypothesis that adolescent binge-drinking impacts the brain and behavior in a manner that persists into adulthood.

Human adolescents drink mostly in a unique binge drinking 'on-off-on' intermittent temporal pattern, drinking at the end of the week and on weekends and abstaining during the first 3-4 days of the week (Zou and Crews, 2012). Daily and intermittent ethanol treatments can have different effects on brain function (Breese et al., 2005). In this study we used an adolescent intermittent ethanol (AIE) treatment ( $5 \mathrm{gm} / \mathrm{kg}$, i.g., 2 days on- 2 days off) designed to model the weekend binge drinking pattern of human adolescents. To assess the persistence of effects following AIE treatment animals remained abstinent during 2 months of maturation into adulthood. We report here that AIE treatment of adolescent rats disrupts adult Barnes Maze reversal learning, but not initial learning acquisition (i.e., the learning 
acquisition curve),. Also, AIE increased specific brain regional volumes determined by magnetic resonance imaging (MRI) and increases expression of extracellular matrix proteins in the orbital frontal cortex (OFC).

\subsection{Material and methods}

\subsubsection{Animal treatment}

Male C57BL/6 mice were ordered from Charles River Labs (Raleigh, NC), and were allowed to acclimate to the animal facilities for seven days in our animal facility prior to treatment. Adolescent mice were requested with the stipulation that all mice were the same weight, in order to reduce potential variability in brain size. Mice were given either water $(\mathrm{N}=7)$ or ethanol $(5 \mathrm{~g} / \mathrm{kg}$, i.g. $25 \%$ ethanol w/v, $\mathrm{N}=8)$ once a day during adolescence on post natal day (P)28-37 in an intermittent fashion (Figure 1).

Either water or ethanol was administered by intragastric (i.g.) administration on days P28, P29, P32, P33, P36, and P37. In our lab this concentration of ethanol given to adolescent mice results in average blood alcohol levels of $310 \mathrm{mg} / \mathrm{dL} \pm 93.7$ (mean $\pm \mathrm{SEM}$ ) measured 1 hour after ethanol treatment. This is a very high binge drinking blood level. There were no differences in body weight between the two groups during the ethanol treatment (Supplemental Figure 1). All protocols were approved by the University of North Carolina Institutional Animal Care and Use Committee and were in accordance with the Congressional Animal Welfare Act.

\subsubsection{Adult Behavior}

Behavioral testing began in young adulthood on P60. The following tests were performed: open field locomotion with center time assessment (P60), learning acquisition using the Barnes Maze at P68 (30 days after the last dose of ethanol), reversal learning using the Barnes Maze (P81-91), and forced swim test P104. Mice were sacrificed by perfusion on P110 for MRI. All behavioral testing was performed in the UNC Neurodevelopmental Disorders Research Center Mouse Behavioral Phenotyping Core using previously published methods (Al-Saffar et al., 2013).

Locomotor activity-Mice spent two hours in the open field chamber $(40 \mathrm{~cm} \times 40 \mathrm{~cm} \times$ $30 \mathrm{~cm}$, VersaMax system, AccuScan Instruments) (Al-Saffar et al., 2013). Total locomotor activity and time spent in the center of the open field were measured.

\subsubsection{Barnes Maze spatial learning with reversal}

The Barnes Maze is a large, brightly-lit, circular platform (diameter $=122 \mathrm{~cm})$, elevated $96.5 \mathrm{~cm}$ from the floor and positioned like a table, with 40 holes (diameter $=5 \mathrm{~cm}$ ) drilled along the perimeter (Figure 2C). An escape box was located under one of the holes, and mice learned which hole allowed for escape from the maze surface. Each mouse was given his own individual escape location, which remained constant across the learning days. Mice were given one trial per day, with up to five minutes per trial to find the escape. The experimenter was blinded to treatment condition. Once criterion for initial learning was achieved ( $>20$ second average latency to escape across all subjects), mice were given four days to rest prior to reversal learning trials. For reversal learning, each mouse's escape location was relocated to the hole that was exactly 180 degrees from the initial location. Mice were then given one 5 minute trial per day until the 20 second average latency was achieved across all subjects. Learning curves are described by the equation $T_{n}=T_{1} n^{b}$, where $T$ is the time to find the correct target, $n$ is the testing day, and $b$ equals $\ln$ (learning ratio)/ $\ln 2$. The learning curve percentage (learning ratio $\times 100 \%$ ) describes how quickly each group learns the task. 


\subsubsection{Forced swim test}

This test was used to assess depressive-like behavior. Mice were placed in a clear beaker filled with $12-15 \mathrm{~cm}$ of tap water $\left(23-26^{\circ} \mathrm{C}\right)$ for 6 minutes. The amount of time a mouse spent immobile during the final 4 minutes was measured.

\subsubsection{Testosterone measurement}

Quantitative testosterone levels in blood serum were measured from plasma of adult mice (P110) using a Parameter ${ }^{\mathrm{TM}}$ Testosterone Assay (Cat \#KGE010) from R\&D systems, Inc. (Minneapolis MN).

\subsubsection{Perfusion and brain tissue preparation for postmortem MRI and IHC}

Mice were sacrificed by perfusion with contrast agent for postmortem brain imaging. Mice were perfused with $0.1 \mathrm{M}$ phosphate buffered saline (PBS) and the gadoteridol contrast agent Prohance (10:1, v:v, $\left.37^{\circ} \mathrm{C}\right)$ (Bracco Diagnostics, Princeton NJ) followed by a mixture of $4 \%$ PFA and Prohance $\left(10: 1,4^{\circ} \mathrm{C}\right)$, similar to a previous study (Badea et al., 2009). Mouse skulls with the brains intact were stored in a PBS/Prohance solution (100:1) until scanning.

\subsubsection{Postmortem MRI Volume using semi-automatic segmentation}

Whole mouse skulls with brain intact were scanned individually at the UNC Biomedical Research Imaging Center (BRIC) on a 9.4 Tesla Bruker BioSpec spectrometer (Bruker Biospin Inc., Billerica, MA). Total scan time was approximately 15 hours per animal. MRI images $(0.12 \mathrm{~mm} \times 0.12 \mathrm{~mm} \times 0.12 \mathrm{~mm})$ were acquired using the following diffusion weighted 3D RARE sequence: $\mathrm{TR}=0.7 \mathrm{~s}, \mathrm{TE}_{\mathrm{eff}}=23.749 \mathrm{~ms}$, Rare Factor $=3$, RARE echo spacing $=11.3067 \mathrm{~ms}$, diffusion gradient time $d=6 \mathrm{~ms}$, diffusion gradient separation $\mathrm{D}=$ 12.422 , b value $-1600 \mathrm{~s} / \mathrm{mm}^{2}$, matrix size $=200 \times 125 \times 80, F O V=24.0 \mathrm{~mm} \times 15 \mathrm{~mm} \times 9.6 \mathrm{~mm}$. Mean diffusivity images, computed from the reconstructed diffusion tensor data, were registered and brain structures were segmented using an atlas-based automatic segmentation protocol developed at the UNC Neuro Image Research and Analysis Laboratory (NIRAL) (Schoenbaum et al., 2009). Following automatic segmentation, blinded investigators manually segmented selected regions of interest identified from the automatic segmentation, as well as measuring region volume and fractional anisotropy using ITK-SNAPTM software version 1.8. Brain region boundaries were determined using the Brookhaven National Library 3-D MRI Digital Atlas Database of the adult C57BL/6 mouse (www.bnl.gov/ medical/RCIBI/mouse) and the mouse brain atlas (Franklin and Paxinos, 2001).

\subsubsection{Immunohistochemistry}

Immunohistochemistry was performed as described previously (Coleman et al., 2009; Crews et al., 2004). Briefly, four sections $(40 \mu \mathrm{m})$ per animal were used. For WFA and neurocan, saggital sections were used. Coronal sections were used for brevican, so both hemispheres were assessed and averaged for each section. Sections were incubated with $0.6 \% \mathrm{H}_{2} \mathrm{O}_{2}$ to remove endogenous peroxidase activity. Primary antibody incubation was performed overnight at $4^{\circ} \mathrm{C}$. The following primary antibodies and dilutions were used: Choline acetyl transferase (ChAT) 1:500, Millipore, Billerica, MA; GFAP 1:500, DAKO, Carpinteria, CA; Iba-1 1:1000, WAKO, Richmond, VA; biotinylated Wisteria floribunda agglutinin (WFA) 1:100, Sigma, Saint Louis, MO; hyaluronan binding protein (HABP) 1:50, Seikagaku, Abingdon, OX, UK; brevican 1:200 Santa Cruz, Santa Cruz, CA; neurocan 1:100, R\&D system. AF5800); phosphacan 1:1000, Chemicon, Temecula, CA; Tenascin-C 1:100, abcam, Cambridge, MA. The following day sections were incubated with the appropriate secondary antibody ( $1 \mathrm{hr}$ at room temperature, 1:200, Vector) followed by reaction with the avidinbiotin complex (1 hr at room temperature, Vector ABC kit, Vector Laboratories). Immunoreactive (IR) neurons were visualized using nickel-enhanced diaminobenzidine 
(DAB) reaction with nickel enhancement. Cortical brain regions were identified using the mouse brain atlas (Franklin and Paxinos, 2001). The Bioquant Nova Advanced Image Analysis System $^{\text {TM }}$ (R\&M Biometric, Nashville, TN) was used to assess histological area and IR-cell density using as described previously (Coleman et al., 2009; Crews et al., 2004). Images were captured by using an Olympus BX50 Microscope and Sony DXC-390 video camera linked to a desktop computer. We have shown previously that this method results in nearly identical percent changes between treatment and control groups as other unbiased stereological techniques (Crews et al., 2004). Perineuronal nets were quantified at a magnification of $200 \times$ for brevican, neruocan, tenascin-C and WFA, and $600 \times$ for phosphacan staining. The staining density was measured in pixels for the defined area (pixels $/ \mathrm{mm}^{2}$ ).

\subsubsection{Structural volume statistics}

For brain structural volume data, Student's $t$ - tests were performed to identify candidate regions for further manual segmentation correction ( $p<0.05$ for significance). Performing multiple comparisons can result in the reporting of false positives, therefore, a false discovery rate (FDR) is necessary to estimate the number of false positives detected by chance. The $q$-value, an FDR method initially introduced by Storey and Tibshirani was employed (He and Lennox, 1947; Isenberg-Grzeda et al., 2012). A $q$-value is similar to a $p$ value in that both values report the minimum false positive rate that is accepted when a test is considered significant. A $q$-value, however, is used when performing multiple comparisons, whereas a $p$-value is most accurate when performing single comparisons. The $q$-values were calculated with $Q$-value module in the $R$ for Windows Software $\odot$ using the bootstrap method (R Development Core Team 1995-2009). Common accepted practice is a threshold of 0.10 (i.e. a $10 \%$ false discovery rate) for the $q$-value. The number of expected false positives is calculated using the formula: $=q_{\text {threshold }} \mathrm{x}$ (\# of data points with $q_{\text {calculated }}$ $<q_{\text {threshold }}$ ). For this MRI brain volume data, a total of less than 0.05 false positives is expected according to the calculation: $0.10\left[q_{\text {threshold }}\right]$ x 5 [of data points with $q_{\text {calculated }}<$ $q_{\text {threshold }}$ = 0.05 [\# false positives]. This shows that it is unlikely that the reported brain volume changes were observed by chance.

When assessing individual treatment groups, the Grubb's Test was employed to identify outliers. A Z score was assigned for each data point: $Z=\mid$ mean-value//standard deviation. The $Z_{\text {critical }}$ was obtained from a Grubb's table of $Z$ scores. A data point with a $Z$ score $>Z_{\text {critical }}$ is considered an outlier and removed from the analysis. No more than one outlier per data set is permitted to be removed. One outlier was detected and removed from the AIE treated group-OFC region $\left(3.78 \mathrm{~mm}^{3}, \mathrm{Z}=2.26, \mathrm{Z}_{\text {critical }}=2.13\right)$.

\subsubsection{Behavioral and Immunohistochemistry statistics}

A 2 way repeated measures ANOVA was performed for learning studies in the Barnes Maze as well as locomotor activity, followed by a Bonferroni post-test to evaluate significance at individual time points.

\subsection{Results}

\subsubsection{AIE causes a reversal learning deficit in adulthood without affecting initial learning}

In previous studies we have found that chronic binge ethanol treatment of rats (Obernier et al., 2002) or adolescent mice (Coleman et al., 2011) do not effect spatial learning in Morris Water maze learning tasks, but did disrupt reversal learning (Coleman et al., 2011; Obernier et al., 2002). In this study, we determine the effect of AIE treatment of mice on spatial learning and reversal learning using the Barnes Maze as well as open field behavior. Mice received either intermittent water (controls) or adolescence intermittent ethanol (AIE, P28- 
37, 6 treatments of $5 \mathrm{gm} / \mathrm{kg}$, Figure 1). Following several weeks of abstinence and maturation, young adult mice were tested for locomotion in an open field test including center time, the Persolt Swim test as well as learning and reversal learning of the escape location in the Barnes Maze. There were no significant differences between groups in total locomotion during the two hours of testing in the open field (Figure 2C. (Figure 2C. $F_{1,299}$ $=1.698 p=0.22$ ). Both groups showed the characteristic pattern of high initial levels of locomotion followed by a reduction in activity with increasing time due to habituation to the chamber with AIE animals appearing to show lower activity at later time points (Figure 2). When the time spent in the center was measured, controls spent 18.4 minutes $(15.4 \%$ of the $2 \mathrm{~h}$ period) exploring the center, while mice in the AIE group spent 10.5 minutes (8.7\% of the $2 \mathrm{~h}$ ) in the center (Figure 2D, $* * p<0.005$ ). Although, AIE binge treated mice spent less time exploring the center of the open field box,. The Persolt forced swim test found no differences in float time between treatment groups (Supplemental Figure 2; $t$-test, $p=0.76$ ). Similarly, there were no differences in serum testosterone concentrations in adulthood following AIE between groups (being $8.1 \pm 2.4 \mathrm{ng} / \mathrm{mL}$ for controls and $10.3 \pm 1.2 \mathrm{ng} / \mathrm{mL}$ for the AIE group (mean $+/-\mathrm{SEM}, p=0.43$ ). Thus, AIE reduced open field center time is consistent with increased anxiety-like behavior, but did not change overall locomotion, Persolt swim test freezing and testosterone in adults following AIE.

Studies using the Barnes Maze to assess learning and reversal learning were initiated 30 days after the last dose of ethanol, e.g. P68. Each mouse was assigned its own specific escape location that remained constant during the initial learning days. Both groups of mice successfully learned to find the escape, reaching criterion by the sixth day of testing (Figure $2 \mathrm{~A})$. There was no significant main effect of adolescent treatment on adult escape latency during initial learning (Figure 2A; 2-way repeated measures ANOVA: $F_{1,65}=2.33 ; p=0.15$,). Neither was there an effect of adolescent treatment on the number of errors during initial learning trials (Figure 2B; $\mathrm{F}_{1,65}=1.37, p=0.26$ ). The learning curve percentages were $49 \%$ and $34 \%$ for control and AIE groups respectively. Thus, adults after AIE did not show changes in Barnes Maze spatial learning.

To assess reversal learning, an index of behavioral plasticity and frontal cortex function, following learning acquisition, the escape location for each mouse was changed to the hole located directly across from the initial learning location at P81 (Figure 3). AIE mice were slower to learn the new escape location than controls across the six days (2-way repeated measures ANOVA: $\mathrm{F}_{1,65}=5.03$, ${ }^{*}<<0.05$,). On the first day of reversal testing, AIE adult mice spent more than twice as much time searching for the new escape location (control: 63.6s vs. ethanol: $149.7 \mathrm{~s}, \mathrm{p}<0.05$ Bonferroni post-test), although AIE treated mice did show similar escape times and low error rates after several days of testing. AIE adult mice also made significantly more errors than controls across the reversal learning period $\left(F_{1,65}=8.011\right.$, ${ }^{p}<0.05$ (Figure 3 ). By the third day of testing, AIE mice were closer to controls in the time needed to escape and the number of errors made. However, the distance travelled in search of the escape remained elevated in AIE mice through the fifth day of testing, where AIE mice traveled more than six times farther than controls $(\mathrm{p}<0.05$, Bonferroni post-test). On average, AIE mice traveled 3.4 times farther than controls searching for the escape across the six days of reversal testing $(\mathrm{p}<0.01, \mathrm{~F}=9.137)$. The data indicate for the first time using the Barnes Maze that AIE caused deficits in reversal learning that persist over six weeks (44 days) of abstinence and maturation to young adulthood.

\subsubsection{AIE and MRI Brain Regional volume in adult mice}

We examined the effect of AIE on the volumes of multiple adult brain regions using structural brain imaging at P110, over 8 weeks after the end of AIE treatment. There were no significant differences in total brain volume between groups ( $401.6 \pm 4.25$ vs. $408.2 \pm$ 
$2.64 \mathrm{~mm}^{3}$, controls vs. AIE group mean $\pm \mathrm{SEM} ; p=0.20, t$-test, P80). However, differences were found in specific brain regions. Surprisingly, several brain regions of AIE treated mice were larger. MRI brain regional volumes enlargements were found in the AIE internal capsule $(+10.1 \%, p<0.002 t$-test, $\mathrm{q}=0.02 \mathrm{FDR})$, orbital frontal cortex $(\mathrm{OFC},+4.2 \%$, $p<0.004 ; q=0.028)$, thalamus $(+2.1 \%, p<0.014 ; q=0.071)$, cerebellum $(+4.5 \%, p<0.019, q$ $=0.089)$, and genu of corpus callosum $(+7.1 \%, p<0.03, q=0.089)$ compared to age matched saline controls (Table 1). There was no volume differences found in the rest of the prefrontal cortex, including the medial prefrontal region (not shown). Also the remainder of the corpus callosum and external capsule without the genu showed no significant differences ( $p=0.13$, $\mathrm{q}=0.26$ ). AIE treatment did not alter MRI whole brain volume at P110, although certain brain regions were found to increase in volume.

\subsubsection{AIE increases Adult OFC Extracellular Matrix Proteins}

The persistent Barnes Maze reversal learning deficits reported here as well as those reported earlier (Coleman et al., 2011). As described above we found increased adult volume following AIE. Since intermittent binge ethanol treatments have been shown to cause acute increases in activation of glial cells (Pascual et al., 2007), we hypothesized that increased activation of microglia or astroglia might contribute to the increase in OFC volume. Glial cell content was assessed using cell specific markers for microglia (Iba1) and astrocytes (GFAP). We did not find any changes in either cortical astroglia cell density $(\mathrm{p}=0.56)$ or cortical microglia structure in the orbitofrontal cortex (data not shown). Thus, the glial cellular content of the OFC did not appear to change. Since we did not find significant longterm changes in the cellular structure of the OFC, we next assessed whether AIE altered extracellular components of the OFC.

The extracellular space in mature brain accounts for about $20 \%$ of total brain volume in the mature brain and is filled with multiple proteins that are organized into the extracellular matrix (ECM) (Dityatev et al., 2010). Extracellular matrix (ECM) proteins are actively formed during brain development and contribute to maturation of neurocircuitry through axon guidance and structural stabilization of axons and synapses (Zimmermann and DoursZimmermann, 2008) suggesting OFC ECM may be developing in adolescence and sensitive to binge drinking. Wisteria floribunda agglutinin (WFA) is a lectin that binds to terminal Nacetylgalactosamine residues, a major constituent of multiple ECM molecules including those within perineuronal nets that surround neuronal cell somas, providing a global measure of ECM. Wisteria floribunda agglutin immunoreactivity (WFA+IR) was visibly more intense in OFC of adult P110 AIE animals than controls (Figure 4A). Quantification found that WFA+IR was increased 55\% in OFC (Figure 4B). There are a large number of ECM proteins, prompting us to focus on specific ECM proteins known to undergo large changes in expression during brain maturation (Zimmermann and Dours-Zimmermann, 2008). We found increased expression of brevican (typically a component of the adult ECM) and neurocan-IR (typically a component of the juvenile ECM) following AIE. Brevican+IR labeled perineuronal nets surrounding cell somas (Figure 5A), and was increased 32\% in the AIE group (Figure 5B). Neurocan+IR showed staining of perineuronal nets in the OFC (Figure 6A), with a substantial 105\% increase of neurocan+IR in the AIE animals (Figure 6B). Phosphocan (a predominately adult ECM protein) and HABP-IR were not different between groups (Figure 7A-B), while Tenasin-C-IR (typically a component of the juvenile $\mathrm{ECM})$ found a statistically non-significant increase $(25 \%, p=0.33)$ in the AIE group. These findings are summarized in Table 2. These findings reveal that AIE results in persistent alterations in the adult ECM in OFC. The relatively larger percent compositions of proteins typically associated with the juvenile form of ECM (i.e. neurocan and tenacin C) suggests that AIE may alter the normal developmental transition of ECM protein expression across adolescence. 


\subsection{Discussion}

This study finds that intermittent adolescent binge ethanol causes persistent alterations in adult brain structure and behavior. Using the Barnes spatial learning maze, we found a persistent reversal learning deficit in adult mice over 40 days after abstinence from AIE treatment, without altering initial spatial learning. Reversal learning is a measure of cognitive flexibility in which an initially learned stimulus-reward association is altered, requiring the subject to learn a new association. In the Barnes Maze the reward is escape from a bright light. In our study we did not find differences between controls and AIE treated animals in learning the location of the escape hole. However, during the reversal task we found AIE treated animals took significantly longer to escape and had significantly more learning errors than controls. The Barnes and Morris Water Maze spatial learning and reversal tasks are commonly used to assess behavioral flexibility (Brigman et al., 2010). We have found that binge treatment of adult rats (Obernier et al., 2002) and continuous adolescent mouse alcohol treatment (Coleman et al., 2011) cause Morris Water Maze reversal learning deficits consistent with the Barnes Maze deficits reported here after adolescent intermittent treatment. Interestingly, studies of chronic intermittent ethanol vapor administration to adult mice find reversal learning deficits using the bowl foraging task at 3 days of abstinence but not 10 days of abstinence (Badanich et al., 2011). We found Barnes Maze reversal learning deficits after 44 days of abstinence following adolescent intermittent ethanol treatment. Badanich et al (2011) concluded chronic ethanol induced reversal learning deficits are due to ethanol induced orbital frontal cortex dysfunction. Studies have suggested that the OFC is essential for reversal learning (Murray and Ranganath, 2007) and OFC lesions in the rat disrupt reversal learning in the radial arm maze (Riceberg and Shapiro, 2012). Although Barnes and Morris Water Maze spatial learning and reversal learning have not been studied following OFC lesions, they are commonly used to assess behavioral flexibility (Brigman et al., 2010) that likely involve multiple brain regions. Human alcoholics have deficits in behavioral flexibility (Fortier et al., 2008; Watson et al., 1981; Wood et al., 1986). Poor judgment and maladaptive decision-making are characteristics of addiction that have been associated with loss of behavioral flexibility (Lucantonio et al., 2012). Our finding of persistently decreased adult behavioral flexibility following adolescent alcohol treatment is consistent with persistent psychopathology that could increase risks of addiction.

AIE was also found to cause persistent alterations in brain structure using MRI. These changes might represent either a disruption of normal development or the initiation of an inflammatory reaction. Total whole brain volume was not significantly altered, though five brain regions were larger in volume in adults that underwent AIE during adolescence: the OFC, internal capsule, genu of corpus callosum, thalamus and cerebellum. This was surprising, given that we have previously observed reductions in the adulthood volume of the basal forebrain and olfactory bulb after a continuous daily ethanol exposure (Coleman et al., 2011). However, our observation of a larger OFC in adult male mice that received AIE is consistent with an observation in human male adolescents with alcohol use disorder (AUD) (Medina et al., 2008). Medina et al found that adolescent males (15-17 y/o) with AUD had larger ventrolateral prefrontal cortical volume (which includes the orbitofrontal cortex) than healthy controls $(\approx 10 \%)$ (Medina et al., 2008). Our data suggests that alcohol exposure might be contributing to this increase in volume. Larger regional brain volumes do not imply improved function, but might actually be associated with cognitive dysfunction. For instance, thalamic enlargement has been associated with negative affect including both depression and suicide in humans (Young et al., 2008; Young et al., 2007) and autistic individuals have larger brains. The brain regional volume enlargements found with AIE (6 treatments, 2 days on- 2 off, $\mathrm{P} 28-37,5 \mathrm{~g} / \mathrm{kg} /$ day i.g.) are different from our previous daily 'continuous' ethanol during adolescence model (10 treatments, $\mathrm{P} 28-37,5 \mathrm{~g} / \mathrm{kg} /$ day i.g.). In 
the previous continuous study, we found volume reductions in the olfactory bulb and the basal forebrain region with a corresponding reduction in cholinergic neurons in the $\mathrm{Ch} 4$ region of the basal forebrain (Coleman et al., 2011). In this current study we did not find a change in the volume of the basal forebrain (Table 1). This indicates that reversal learning deficits are consistently found across various adolescent alcohol treatment models, whereas changes in adult MRI brain regional volumes and neurochemistry appear to be dependent upon the quantity and frequency of adolescent binge exposure.

Though AIE caused increases in the volumes of certain brain regions, the mechanism behind this phenomenon is unclear. Although brain volume changes during adolescence, it is not known how rodent brain volume changes during adolescence though adulthood human studies find that the frontal regions of the adolescent cortex undergo a developmental change in volume, resulting inverted U shaped pattern of across adolescence (Giedd, 2004; Giedd et al., 1999; Gogtay et al., 2004; Lenroot and Giedd, 2006; Shaw et al., 2006). Gogtay et al found that the volume of dorsolateral prefrontal cortex, which includes the orbitofrontal cortex (OFC), declines to adult size during adolescence, between ages 16-20 (Gogtay et al., 2004). Factors that could underlie the increases in brain regional volumes include increases in neurons, white matter, neuropil, glia or cerebral edema. We did not find any differences in glia structure or numbers between groups in the adult cortex, suggesting that gliogenesis is not the underlying mechanism. Although we did not assess white matter histologically, it is interesting that the majority of the regions that we found to have larger volumes in AIE treated adults are either purely white matter tracts or contain substantial white matter tracts. White matter maturation in the cortex, cerebellum, limbic regions and internal capsule continues through adolescence into early adulthood (Giedd, 2004; Juraska and Markham, 2004; Ostby et al., 2009; Paus et al., 1999). Growth of white matter includes multiple components, ECM are increased as white matter grows and could contribute to the growth. For example, brevican expression is high in white matter oligodendrocytes during active myelination (Zimmermann and Dours-Zimmermann, 2008). The enlarged ventrolateral prefrontal cortical volume in males with AUD observed by Medina et al was associated with a $7 \%$ increase in white matter. Thus, it is possible that our finding of AIE induced increased in volume of OFC and white matter regions is related to overexpression of ECM. However, it is important to note that studies of normal human aging show reduced frontal cortical volumes with age matched adult alcoholics showing larger reductions typically associated with white matter reductions (Pfefferbaum et al., 2006; Pfefferbaum et al., 1996). Thus, the impact of adolescent binge drinking on brain structural volumes appears to differ from chronic adult alcoholism which is associated with reduced brain volumes.

We focused histology on the OFC due to the link of this brain region to reversal learning and behavioral flexibility. Water makes up about $80 \%$ of brain weight, and binge ethanol treatment of adult rats has been shown to increase brain water content by $1-2 \%$ (Collins et al., 1996). Our finding of a persistent increase in $4 \%$ OFC volume could be related to water content, although changes in water content would be expected to be transient. The extracellular matrix (ECM) contributes to neurophil and is a complex network composed primarily of glycoproteins and proteoglycans linked to a hyaluronan backbone and tenasins (Rauch, 2004; Zimmermann and Dours-Zimmermann, 2008). ECM proteins can aggregate to form perineuronal nets, which can modulate neuron function (Costa et al., 2007). We found increased expression of WFA (terminal $\mathrm{N}$-acetylgalactosamines), brevican and neurocan, all of which are present in perineuronal nets (PNN). PNN form during development around cortical interneurons at a stage of development that coincides with the termination of critical periods of experience dependent brain wiring (Gundelfinger et al., 2010). Removal of PNN restores plasticity in visual cortex and amygdala consistent with PNN reducing plasticity. Brevican and WFA overlap in many PNN with both likely contributing to inhibition of neurite outgrowth and stabilization of neural networks 
(Zimmermann and Dours-Zimmermann, 2008). Neurocan is predominantly a juvenile ECM protein suggesting increased levels of ECM may be related to delayed or altered maturation the ECM. Since formation of ECM regulates neuronal migration, synapse formation, axonal outgrowth and synaptic plasticity (Bandtlow and Zimmermann, 2000; Frischknecht et al., 2009; Gundelfinger et al., 2010), altered ECM maturation would be expected to alter neurocircuitry. Our finding of increased ECM is consistent with reduced plasticity in OFC. Since OFC is important for behavioral flexibility as assessed by reversal learning it is possible that the increase in ECM in OFC contributes to the deficits in reversal learning.

Ethanol treatment has been found to alter ECM in the adult rat hippocampus and prefrontal cortex by reducing the activation of matrix metalloproteinase-9, a protease involved in ECM reorganization (Wright et al., 2003). ECM proteins are key to cortical development and undergo major changes in expression during adolescence, when underage binge drinking peaks. In mice a developmental reorganization of ECM occurs beginning around P20 from a juvenile form consisting primarily of neurocan, versican- $\mathrm{V} 0$ and $\mathrm{V} 1$, and tenasin-C to an adult form consisting mainly of brevican, versican-V2, aggregan, phosophocan, and tenascin-R (Gundelfinger et al., 2010; Zimmermann and Dours-Zimmermann, 2008). Further, ECM reorganization and/or deposition in the cortex is known to increase following acute ethanol treatment (Wright et al., 2003) neurotoxicity, hypoxia, and inflammation (Leonardo et al., 2008; Silver and Miller, 2004). We have found previously that ethanol can acutely increase neuro-inflammation and cause microglia activation (Qin et al., 2008; Zou and Crews, 2010) which could alter ECM reorganization. We observed increased immunohistochemical staining of WFA, brevican, and neurocan, a non-significant trend for tenacin-C and no change in phosphocan and HABP in the OFC (Table 2). Although, there are more ECM proteins to investigate, we found the largest increase in ECM+IR in neurocan, which is found primarily in the juvenile form of the ECM (Gundelfinger et al., 2010; Zimmermann and Dours-Zimmermann, 2008) consistent with AIE altering the developmental transition from juvenile to adult ECM. ECM neuronal nets primarily cover GABAergic neurons and modulate AMPA receptor mediated synaptic plasticity (Celio and Blumcke, 1994; Celio et al., 1998; Frischknecht et al., 2009). Acute ethanol treatment has been shown to cause increased turnover of ECM evidenced by increased matrix metalloproteinase- 9 expression in the prefrontal cortex and hippocampus that corresponded with an impairment spatial learning (Wright et al., 2003). ECM protein expression in the amygdala has also been found to be associated with the maintenance of fear conditioning (Gogolla et al., 2009). We observed that AIE altered ECM protein expression in the adult OFC and caused a reversal learning deficit and decrease time spent in the center of the open field locomotion test. Additional studies are needed to determine if these behavioral changes are linked to the changes in ECM.

\subsection{Conclusion}

This study reveals that a mouse model of adolescent binge drinking has consequences on adult brain structure and behavior. AIE induced structural and behavioral changes in adult mice following the 6 binge ethanol doses of AIE, similar to those found in some human studies. A larger adult OFC (observed in human adolescent binge-drinkers), reversal learning deficits (seen in alcoholics and cocaine abusers) and heightened anxiety-like behavior (seen in alcoholics) were found in adult mice that underwent AIE. This suggests that individuals who participate in episodic binge drinking during adolescence may be at risk for brain structural alterations, reversal learning deficits, and heightened anxiety that can persist for years after binge-drinking. These behavioral dysfunctions could put individuals at risk for numerous difficulties in adult life. We evaluated the ECM in the adult OFC due to the role of the OFC in reversal learning and due to our current adult MRI brain volume findings following AIE. To our knowledge, no group has previously characterized ECM 
protein expression in the OFC. We report that an adolescent intermittent ethanol binge model results in alterations in adulthood including: specific brain structural volume changes, alterations in the ECM protein expression in the OFC, a reversal learning deficit, and increased anxiety-like behavior.

\section{Supplementary Material}

Refer to Web version on PubMed Central for supplementary material.

\section{Acknowledgments}

The authors wish to thank the National Institute on Alcohol Abuse and Alcoholism (AA06069; AA011605, AA018051, AA20022, AA20023), the UNC-Bowles Center for Alcohol Studies and the UNC Neurodevelopmental Disorders Research Center (NINDS R42 NS059095, NIDA P01 DA022446-01, NIMH R41 NS059095) for support.

\section{References}

Al-Saffar H, Lewis K, Liu E, Schober A, Corrigan JJ, Shibata K, Steiner AA. Lipopolysaccharideinduced hypothermia and hypotension are associated with inflammatory signaling that is triggered outside the brain. Brain, behavior, and immunity. 2013; 28:188-195.

Badanich KA, Becker HC, Woodward JJ. Effects of chronic intermittent ethanol exposure on orbitofrontal and medial prefrontal cortex-dependent behaviors in mice. Behavioral Neuroscience. 2011; 125(6):879-891. [PubMed: 22122149]

Badea A, Johnson GA, Williams RW. Genetic dissection of the mouse brain using high-field magnetic resonance microscopy. Neuroimage. 2009; 45(4):1067-1079. [PubMed: 19349225]

Bandtlow CE, Zimmermann DR. Proteoglycans in the developing brain: new conceptual insights for old proteins. Physiological Reviews. 2000; 80(4):1267-1290. [PubMed: 11015614]

Basak R, Bandyopadhyay R. Encapsulation of hydrophobic drugs in Pluronic F127 micelles: effects of drug hydrophobicity, solution temperature, and $\mathrm{pH}$. Langmuir : the ACS journal of surfaces and colloids. 2013; 29(13):4350-4356. [PubMed: 23472840]

Breese GR, Overstreet DH, Knapp DJ. Conceptual framework for the etiology of alcoholism: a "kindling"/stress hypothesis. Psychopharmacology (Berl). 2005; 178(4):367-380. [PubMed: 15765253]

Brigman JL, Graybeal C, Holmes A. Predictably irrational: assaying cognitive inflexibility in mouse models of schizophrenia. Frontiers in neuroscience. 2010; 4

Brown SA, Tapert SF, Granholm E, Delis DC. Neurocognitive functioning of adolescents: effects of protracted alcohol use. Alcoholism, clinical and experimental research. 2000; 24(2):164-171.

Celio MR, Blumcke I. Perineuronal nets--a specialized form of extracellular matrix in the adult nervous system. Brain research. Brain research reviews. 1994; 19(1):128-145. [PubMed: 8167657]

Celio MR, Spreafico R, De Biasi S, Vitellaro-Zuccarello L. Perineuronal nets: past and present. Trends in neurosciences. 1998; 21(12):510-515. [PubMed: 9881847]

Coleman LG Jr, He J, Lee J, Styner M, Crews FT. Adolescent binge drinking alters adult brain neurotransmitter gene expression, behavior, brain regional volumes, and neurochemistry in mice. Alcohol Clin Exp Res. 2011; 35(4):671-688. [PubMed: 21223304]

Coleman LG Jr, Jarskog LF, Moy SS, Crews FT. Deficits in adult prefrontal cortex neurons and behavior following early post-natal NMDA antagonist treatment. Pharmacol Biochem Behav. 2009; 93(3):322-330. [PubMed: 19409920]

Collins MA, Corso TD, Neafsey EJ. Neuronal degeneration in rat cerebrocortical and olfactory regions during subchronic "binge" intoxication with ethanol: possible explanation for olfactory deficits in alcoholics. Alcoholism: Clinical and Experimental Research. 1996; 20(2):284-292.

Costa C, Tortosa R, Domenech A, Vidal E, Pumarola M, Bassols A. Mapping of aggrecan, hyaluronic acid, heparan sulphate proteoglycans and aquaporin 4 in the central nervous system of the mouse. Journal of chemical neuroanatomy. 2007; 33(3):111-123. [PubMed: 17349777] 
Crews F, He J, Hodge C. Adolescent cortical development: a critical period of vulnerability for addiction. Pharmacol Biochem Behav. 2007; 86(2):189-199. [PubMed: 17222895]

Crews FT, Braun CJ, Hoplight B, Switzer RC 3rd, Knapp DJ. Binge ethanol consumption causes differential brain damage in young adolescent rats compared with adult rats. Alcoholism, clinical and experimental research. 2000; 24(11):1712-1723.

Crews FT, Nixon K, Wilkie ME. Exercise reverses ethanol inhibition of neural stem cell proliferation. Alcohol. 2004; 33(1):63-71. [PubMed: 15353174]

Dityatev A, Seidenbecher CI, Schachner M. Compartmentalization from the outside: the extracellular matrix and functional microdomains in the brain. Trends Neurosci. 2010; 33(11):503-512. [PubMed: 20832873]

Ehlers CL, Criado JR, Wills DN, Liu W, Crews FT. Periadolescent ethanol exposure reduces adult forebrain ChAT+IR neurons: correlation with behavioral pathology. Neuroscience. 2011; 199:333-345. [PubMed: 22033458]

Escobar-Chavez JJ, Lopez-Cervantes M, Naik A, Kalia YN, Quintanar-Guerrero D, Ganem-Quintanar A. Applications of thermo-reversible pluronic F-127 gels in pharmaceutical formulations. Journal of pharmacy \& pharmaceutical sciences : a publication of the Canadian Society for Pharmaceutical Sciences, Societe canadienne des sciences pharmaceutiques. 2006; 9(3):339-358.

Fabio MC, Nizhnikov ME, Spear NE, Pautassi RM. Binge ethanol intoxication heightens subsequent ethanol intake in adolescent, but not adult, rats. Developmental psychobiology. 2013

Fortier CB, Steffen EM, Lafleche G, Venne JR, Disterhoft JF, McGlinchey RE. Delay discrimination and reversal eyeblink classical conditioning in abstinent chronic alcoholics. Neuropsychology. 2008; 22(2):196-208. [PubMed: 18331162]

Franklin, Paxinos. The Mouse Brain in Stereotaxic Coordinates. 2001

Frischknecht R, Heine M, Perrais D, Seidenbecher CI, Choquet D, Gundelfinger ED. Brain extracellular matrix affects AMPA receptor lateral mobility and short-term synaptic plasticity. Nature Neuroscience. 2009; 12(7):897-904.

Giedd JN. Structural magnetic resonance imaging of the adolescent brain. Ann N Y Acad Sci. 2004; 1021:77-85. [PubMed: 15251877]

Giedd JN, Blumenthal J, Jeffries NO, Castellanos FX, Liu H, Zijdenbos A, Paus T, Evans AC, Rapoport JL. Brain development during childhood and adolescence: a longitudinal MRI study. Nature Neuroscience. 1999; 2(10):861-863.

Gogolla N, Caroni P, Luthi A, Herry C. Perineuronal nets protect fear memories from erasure. Science. 2009; 325(5945):1258-1261. [PubMed: 19729657]

Gogtay N, Giedd JN, Lusk L, Hayashi KM, Greenstein D, Vaituzis AC, Nugent TF 3rd, Herman DH, Clasen LS, Toga AW, Rapoport JL, Thompson PM. Dynamic mapping of human cortical development during childhood through early adulthood. Proc Natl Acad Sci U S A. 2004; 101(21): 8174-8179. [PubMed: 15148381]

Gong G, Yuan LB, Hu L, Wu W, Yin L, Hou JL, Liu YH, Zhou LS. Glycyrrhizin attenuates rat ischemic spinal cord injury by suppressing inflammatory cytokines and HMGB1. Acta pharmacologica Sinica. 2012; 33(1):11-18. [PubMed: 22158106]

Gruber E, DiClemente RJ, Anderson MM, Lodico M. Early drinking onset and its association with alcohol use and problem behavior in late adolescence. Preventive medicine. 1996; 25(3):293-300. [PubMed: 8781007]

Gundelfinger ED, Frischknecht R, Choquet D, Heine M. Converting juvenile into adult plasticity: a role for the brain's extracellular matrix. The European journal of neuroscience. 2010; 31(12): 2156-2165. [PubMed: 20497467]

Hanson KL, Medina KL, Padula CB, Tapert SF, Brown SA. Impact of Adolescent Alcohol and Drug Use on Neuropsychological Functioning in Young Adulthood: 10-Year Outcomes. Journal of child $\&$ adolescent substance abuse. 2011; 20(2):135-154. [PubMed: 21532924]

He DW, Lennox B. Cerebral beriberi (Wernicke's encephalopathy); review of 52 cases in a Singapore prisoner-of-war hospital. Lancet. 1947; 1(6436):11-17. [PubMed: 20278697]

Hingson R, Winter M. Epidemiology and consequences of drinking and driving. Alcohol research \& health : the journal of the National Institute on Alcohol Abuse and Alcoholism. 2003; 27(1):63-78. [PubMed: 15301401] 
Isenberg-Grzeda E, Kutner HE, Nicolson SE. Wernicke-Korsakoff-syndrome: under-recognized and under-treated. Psychosomatics. 2012; 53(6):507-516. [PubMed: 23157990]

Johnston, L.; O'Malley, P.; Bachman, J.; Schulenberg, JE. Seconday school students, 2008. Vol. Vol.1. Bethesda, MD: National Institute on Drug Abuse; 2009. Monitoring the future: National survey results on drug use, 1975-2008. xxxiv

Juraska JM, Markham JA. The cellular basis for volume changes in the rat cortex during puberty: white and gray matter. Annals of the New York Academy of Sciences. 2004; 1021:431-435. [PubMed: 15251923]

Lenroot RK, Giedd JN. Brain development in children and adolescents: insights from anatomical magnetic resonance imaging. Neuroscience and biobehavioral reviews. 2006; 30(6):718-729. [PubMed: 16887188]

Leonardo CC, Eakin AK, Ajmo JM, Gottschall PE. Versican and brevican are expressed with distinct pathology in neonatal hypoxic-ischemic injury. Journal of neuroscience research. 2008; 86(5): 1106-1114. [PubMed: 17972319]

Lucantonio F, Stalnaker TA, Shaham Y, Niv Y, Schoenbaum G. The impact of orbitofrontal dysfunction on cocaine addiction. Nature Neuroscience. 2012; 15(3):358-366.

Medina KL, McQueeny T, Nagel BJ, Hanson KL, Schweinsburg AD, Tapert SF. Prefrontal cortex volumes in adolescents with alcohol use disorders: unique gender effects. Alcoholism, clinical and experimental research. 2008; 32(3):386-394.

Murray LJ, Ranganath C. The dorsolateral prefrontal cortex contributes to successful relational memory encoding. The Journal of neuroscience : the official journal of the Society for Neuroscience. 2007; 27(20):5515-5522. [PubMed: 17507573]

O'Malley PM, Johnston LD, Bachman JG. Alcohol use among adolescents. Alcohol health and research world. 1998; 22(2):85-93. [PubMed: 15706782]

Obernier JA, White AM, Swartzwelder HS, Crews FT. Cognitive deficits and CNS damage after a 4day binge ethanol exposure in rats. Pharmacol Biochem Behav. 2002; 72(3):521-532. [PubMed: 12175448]

Ostby Y, Tamnes CK, Fjell AM, Westlye LT, Due-Tonnessen P, Walhovd KB. Heterogeneity in subcortical brain development: A structural magnetic resonance imaging study of brain maturation from 8 to 30 years. The Journal of neuroscience : the official journal of the Society for Neuroscience. 2009; 29(38):11772-11782. [PubMed: 19776264]

Pascual M, Blanco AM, Cauli O, Minarro J, Guerri C. Intermittent ethanol exposure induces inflammatory brain damage and causes long-term behavioural alterations in adolescent rats. Eur $\mathbf{J}$ Neurosci. 2007; 25(2):541-550. [PubMed: 17284196]

Paus T, Zijdenbos A, Worsley K, Collins DL, Blumenthal J, Giedd JN, Rapoport JL, Evans AC. Structural maturation of neural pathways in children and adolescents: in vivo study. Science. 1999; 283(5409):1908-1911. [PubMed: 10082463]

Pfefferbaum A, Adalsteinsson E, Sullivan EV. Dysmorphology and microstructural degradation of the corpus callosum: Interaction of age and alcoholism. Neurobiology of aging. 2006; 27(7):9941009. [PubMed: 15964101]

Pfefferbaum A, Lim KO, Desmond JE, Sullivan EV. Thinning of the corpus callosum in older alcoholic men: A magnetic resonance imaging study. Alcoholism, Clinical and Experimental Research. 1996; 20:752-757.

Qin L, He J, Hanes RN, Pluzarev O, Hong JS, Crews FT. Increased systemic and brain cytokine production and neuroinflammation by endotoxin following ethanol treatment. $\mathrm{J}$ Neuroinflammation. 2008; 5:10. [PubMed: 18348728]

Qin L, Liu Y, Hong JS, Crews FT. NADPH oxidase and aging drive microglial activation, oxidative stress, and dopaminergic neurodegeneration following systemic LPS administration. Glia. 2013; 61(6):855-868. [PubMed: 23536230]

Rauch U. Extracellular matrix components associated with remodeling processes in brain. Cellular and molecular life sciences : CMLS. 2004; 61(16):2031-2045. [PubMed: 15316653]

Riceberg JS, Shapiro ML. Reward stability determines the contribution of orbitofrontal cortex to adaptive behavior. The Journal of neuroscience : the official journal of the Society for Neuroscience. 2012; 32(46):16402-16409. [PubMed: 23152622] 
SAMSA. Substance Abuse and Mental Health Services Administration. (2007). Rockville, MD: Results from the 2006 National Survey on Drug Use and Health: National Findings (Office of Applied Studies, NSDUH Series H-32, DHHS Publication No. SMA 07-4293); 2007.

Schoenbaum G, Roesch MR, Stalnaker TA, Takahashi YK. A new perspective on the role of the orbitofrontal cortex in adaptive behaviour. Nature reviews. Neuroscience. 2009; 10(12):885-892.

Sharma PK, Bhatia SR. Effect of anti-inflammatories on Pluronic F127: micellar assembly, gelation and partitioning. International journal of pharmaceutics. 2004; 278(2):361-377. [PubMed: 15196641]

Shaw P, Greenstein D, Lerch J, Clasen L, Lenroot R, Gogtay N, Evans A, Rapoport J, Giedd J. Intellectual ability and cortical development in children and adolescents. Nature. 2006; 440(7084): 676-679. [PubMed: 16572172]

Silver J, Miller JH. Regeneration beyond the glial scar. Nature reviews. Neuroscience. 2004; 5(2):146156.

Spear LP. The adolescent brain and age-related behavioral manifestations. Neurosci Biobehav Rev. 2000; 24(4):417-463. [PubMed: 10817843]

Tapert SF, Brown SA. Substance dependence, family history of alcohol dependence and neuropsychological functioning in adolescence. Addiction. 2000; 95(7):1043-1053. [PubMed: 10962769]

Torchilin VP. Structure and design of polymeric surfactant-based drug delivery systems. Journal of controlled release : official journal of the Controlled Release Society. 2001; 73(2-3):137-172. [PubMed: 11516494]

Watson AJ, Walker JF, Tomkin GH, Finn MM, Keogh JA. Acute Wernickes encephalopathy precipitated by glucose loading. Irish journal of medical science. 1981; 150(10):301-303. [PubMed: 7319764]

Wechsler H, Dowdall GW, Davenport A, Castillo S. Correlates of college student binge drinking. American journal of public health. 1995; 85(7):921-926. [PubMed: 7604914]

White AM, Hingson RW, Pan IJ, Yi HY. Hospitalizations for alcohol and drug overdoses in young adults ages 18-24 in the United States, 1999-2008: results from the Nationwide Inpatient Sample. Journal of studies on alcohol and drugs. 2011; 72(5):774-786. [PubMed: 21906505]

White HR, Marmorstein NR, Crews FT, Bates ME, Mun EY, Loeber R. Associations between heavy drinking and changes in impulsive behavior among adolescent boys. Alcoholism, clinical and experimental research. 2011; 35(2):295-303.

Wood B, Currie J, Breen K. Wernicke's encephalopathy in a metropolitan hospital. A prospective study of incidence, characteristics and outcome. The Medical Journal of Australia. 1986; 144(1): 12-16. [PubMed: 3941614]

Wright JW, Masino AJ, Reichert JR, Turner GD, Meighan SE, Meighan PC, Harding JW. Ethanolinduced impairment of spatial memory and brain matrix metalloproteinases. Brain Research. 2003; 963(1-2):252-261. [PubMed: 12560131]

Young KA, Bonkale WL, Holcomb LA, Hicks PB, German DC. Major depression, 5HTTLPR genotype, suicide and antidepressant influences on thalamic volume. The British journal of psychiatry : the journal of mental science. 2008; 192(4):285-289. [PubMed: 18378990]

Young KA, Holcomb LA, Bonkale WL, Hicks PB, Yazdani U, German DC. 5HTTLPR polymorphism and enlargement of the pulvinar: unlocking the backdoor to the limbic system. Biological Psychiatry. 2007; 61(6):813-818. [PubMed: 17083920]

Zhu S, Li W, Ward MF, Sama AE, Wang H. High mobility group box 1 protein as a potential drug target for infection- and injury-elicited inflammation. Inflammation \& allergy drug targets. 2010; 9(1):60-72. [PubMed: 19906009]

Zimmermann DR, Dours-Zimmermann MT. Extracellular matrix of the central nervous system: from neglect to challenge. Histochemistry and cell biology. 2008; 130(4):635-653. [PubMed: 18696101]

Zou J, Crews F. Induction of innate immune gene expression cascades in brain slice cultures by ethanol: key role of NF-kappaB and proinflammatory cytokines. Alcohol Clin Exp Res. 2010; 34(5):777-789. [PubMed: 20201932] 
Zou J, Crews FT. Inflammasome-IL-1beta Signaling Mediates Ethanol Inhibition of Hippocampal Neurogenesis. Frontiers in neuroscience. 2012; 6:77. [PubMed: 22661925] 


\section{Highlights}

- A rat adolescent intermittent ethanol (AIE) binge model assessed in adult brain.

- AIE did not change learning, but caused reversal learning deficits.

- Orbital frontal cortex (OFC) brain volume was increased following AIE.

- AIE increased OFC expression of extracellular matrix proteins. 


\section{A. Adolescent Intermittent Ethanol Binge}

Adult Testing

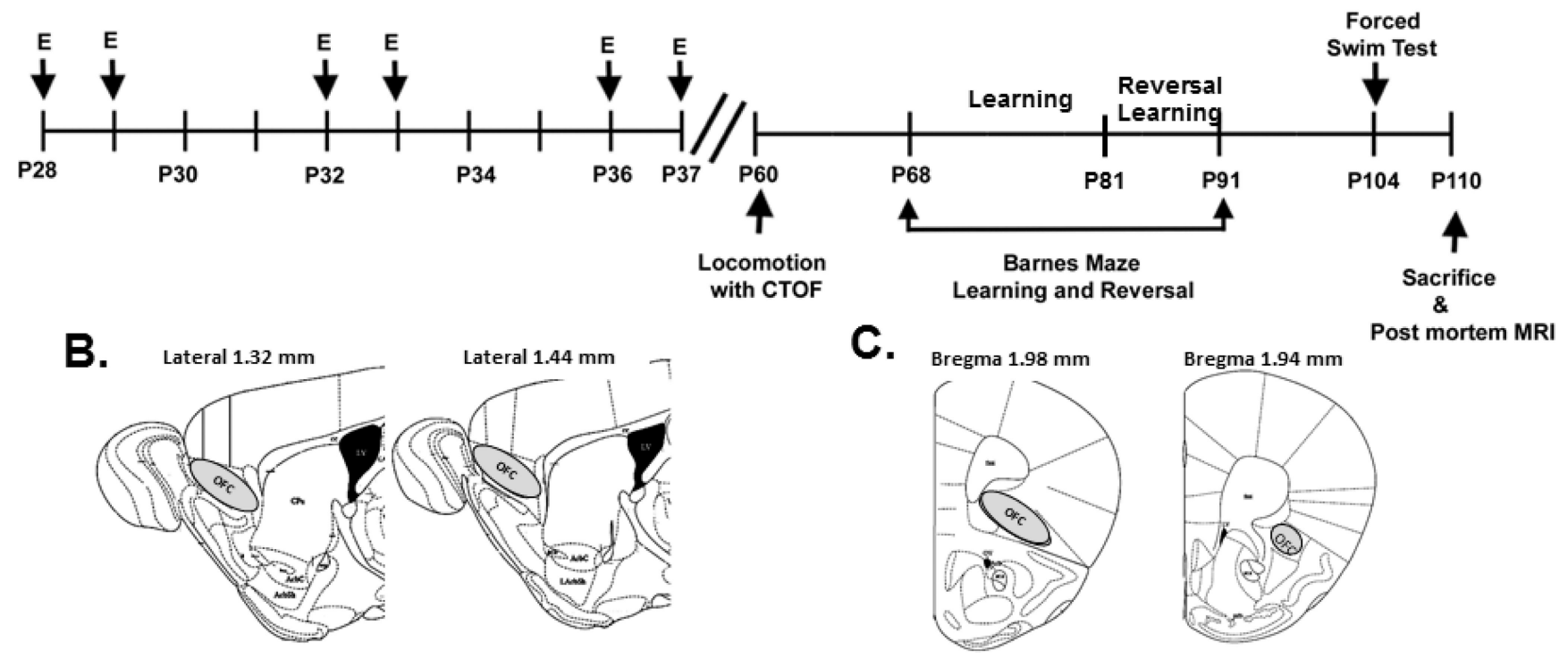

Figure 1.

Adolescent intermittent ethanol (AIE) binge and adult behavioral testing schedules. Mice were given either water or ethanol $(5 \mathrm{~g} / \mathrm{kg}$, i.g. $25 \%$ ethanol w/v) once a day during adolescence (P28-37) in an intermittent fashion. Water or ethanol was administered on days P28, P29, P32, P33, P36, and P37. Behavioral testing began in young adulthood on P60. The following tests were performed: open field locomotion with center time (P60), Studies using the Barnes Maze to assess learning started at P68, 30 days after the last dose of ethanol. Reversal learning studies were P81-91 and forced swim test P104. Mice were sacrificed by perfusion on P110 for MRI. Following MRI scans using the intact head, the brain was removed and sectioned for immunohistochemistry either sagittal (B) or coronal (C). Coronal sections were used for brevican as shown above. To better localize OFC we shifted to sagittal sections for WFA, neurocan, HABP, Phosphocan and Tenacin-C. 
Initial Learning Latency to Escape In Adults

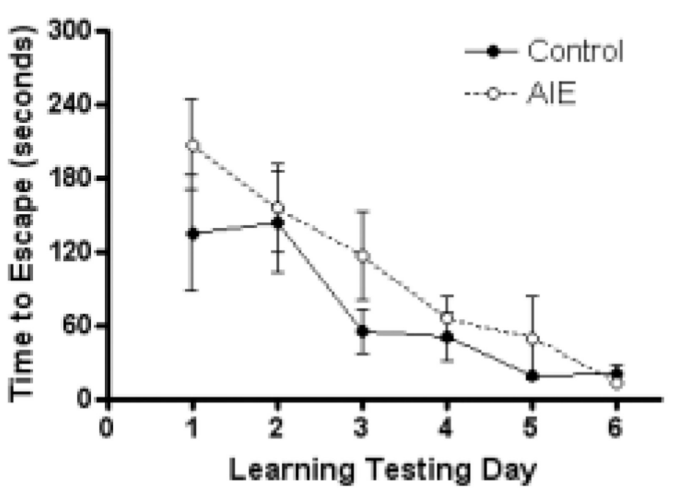

B

\section{C}

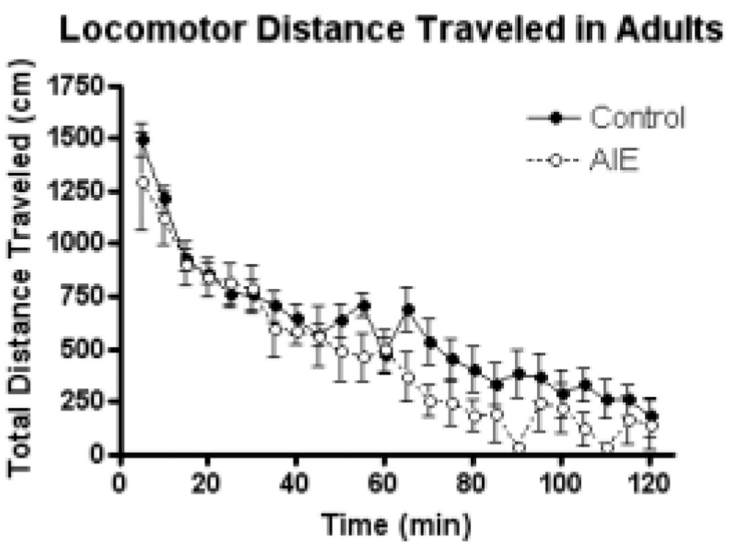

D

Initial Learning Errors in Adults

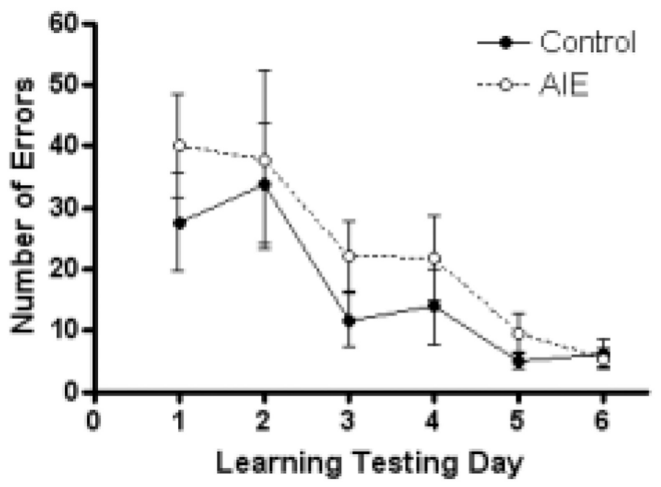

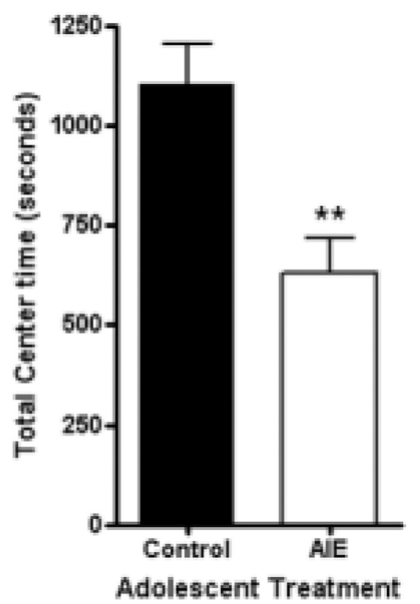

\section{Center Time in Open Field in Adults}

Figure 2.

The effects of adolescent intermittent ethanol (AIE) on spatial learning and unconditioned anxiety-like behavior. Mice were given either water or ethanol $(5 \mathrm{~g} / \mathrm{kg}$, i.g.) in an intermittent fashion ( 2 days on -2 days off) during adolescence (P28-37). (A-B) AIE did not alter initial spatial learning in adult mice. Spatial learning was assessed using the Barnes maze across six days in adulthood (P68-73). (A) Latency to escape. There was no significant main effect of adolescent treatment on latency to find the escape location during initial learning days (2-way ANOVA: $\mathrm{p}=0.15, \mathrm{~F}_{1,65}=2.33$ ). (B) Errors during initial learning. There was no significant main effect of adolescent treatment on the number of errors made during initial learning $\left(\mathrm{p}=0.26, \mathrm{~F}_{1,65}=1.37\right)$. (C-D) AIE mice showed increased unconditioned anxietylike behavior in adulthood. Total locomotor activity and time spent in the center of the open field (CTOF) were assessed in young adults (P60). (C) Total locomotor activity. AIE did not alter total locomotor distance travelled in adult mice across the two hour testing period $\left(\mathrm{p}=0.22, \mathrm{~F}_{1,299}=1.70\right)$. (D) CTOF. Mice that underwent AIE binge spent less time in the center of the open field than controls (57\% of controls, ${ }^{* *} \mathrm{p}<0.005$ ), consistent with increased unconditioned anxiety-like behavior. $\mathrm{N}=7$ Control, 8 AIE mice. 


\section{Control}
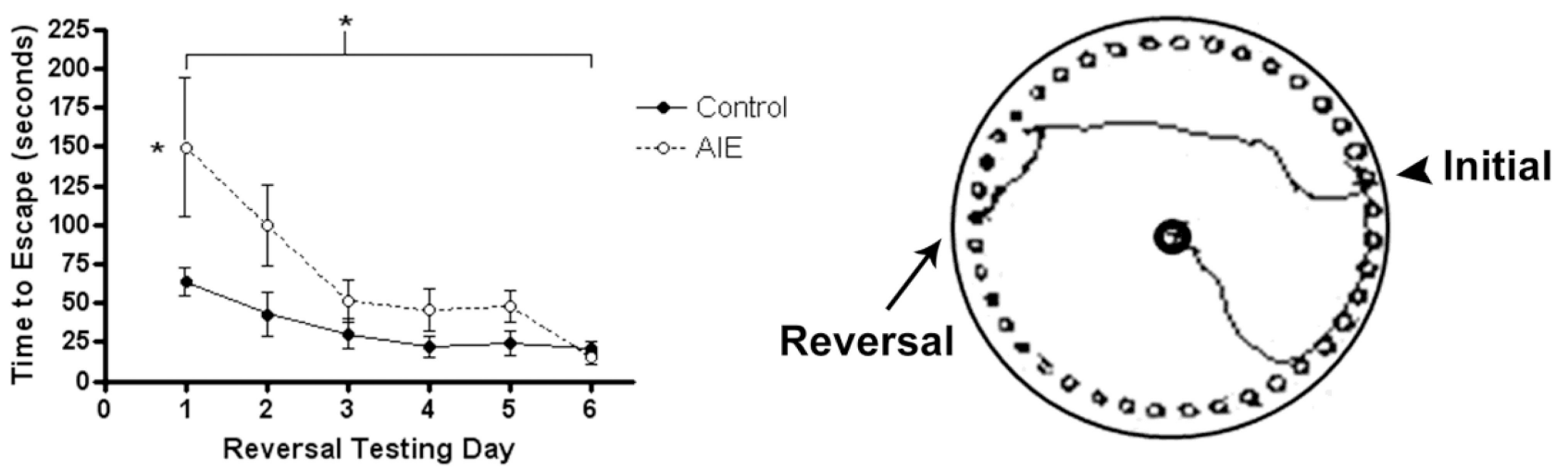

B

\section{Reversal Learning Errors in Adults}

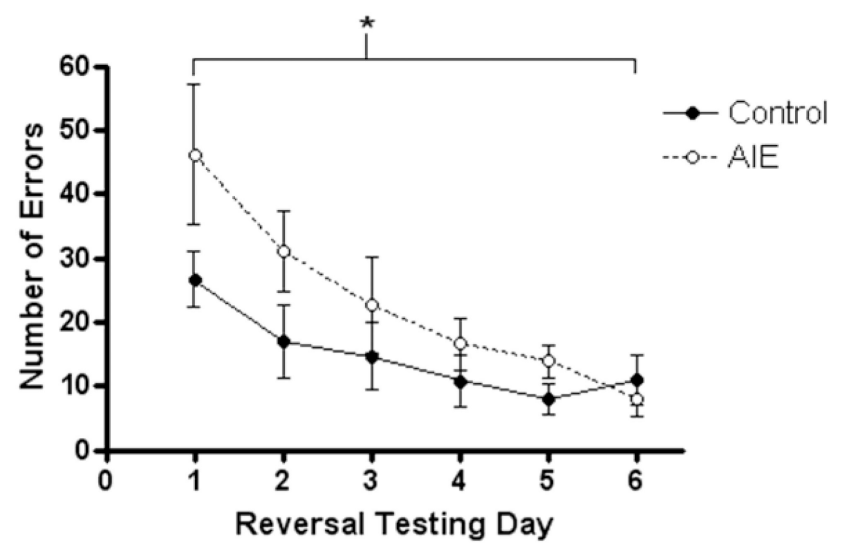

Adolescent IE

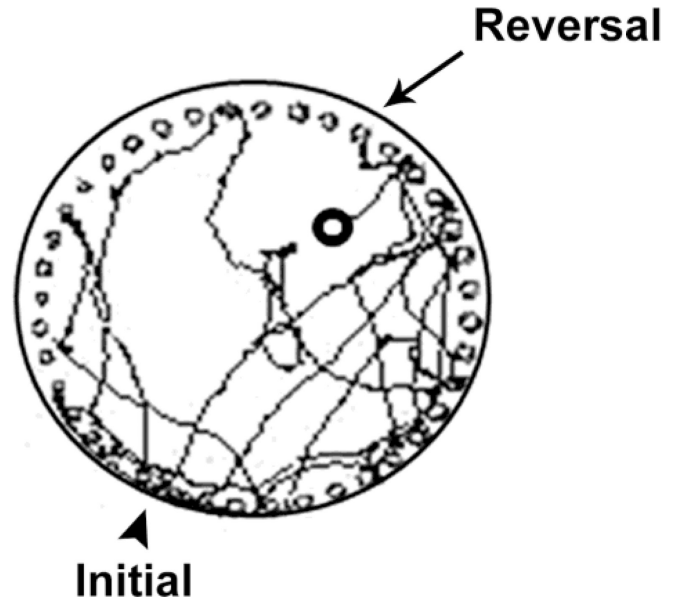

Figure 3.

Adolescent intermittent ethanol (AIE) binge caused a reversal learning deficit in adulthood. Mice were given either water or ethanol $(5 \mathrm{~g} / \mathrm{kg}$, i.g.) in an intermittent fashion (2 days on 2 days off) during adolescence (P28-37). Reversal learning using the Barnes maze was assessed beginning on P81 across six days following the acquisition of initial spatial learning (P68-73). The escape location for each mouse was moved 180 degrees from its location during the acquisition of initial learning. (A) Latency to escape. AIE mice were slower to learn the new escape location than controls across the six days (2-way repeated measures ANOVA: $\mathrm{F}_{1,65}=5.03$, * $\left.\mathrm{p}<0.05\right)$, with AIE adult mice spending more than twice as much time searching for the new escape on the first day of reversal testing than controls $(* \mathrm{p}<0.05$, Bonferroni post-test).

(B) Errors during reversal learning. AIE adult mice also made significantly more errors than controls across the reversal learning period $\left(\mathrm{F}_{1,65}=8.011,{ }^{*} \mathrm{p}<0.05\right)$.

(C) Tracings of the paths taken by one control and one AIE mouse in search of the escape on reversal day 1 . Note how both mice revisited the initial learning location, though the AIE mouse searched more at the former and traveled substantially more around the entire maze than the control mouse. $\mathrm{N}=7$ Control, 8 AIE mice 
A
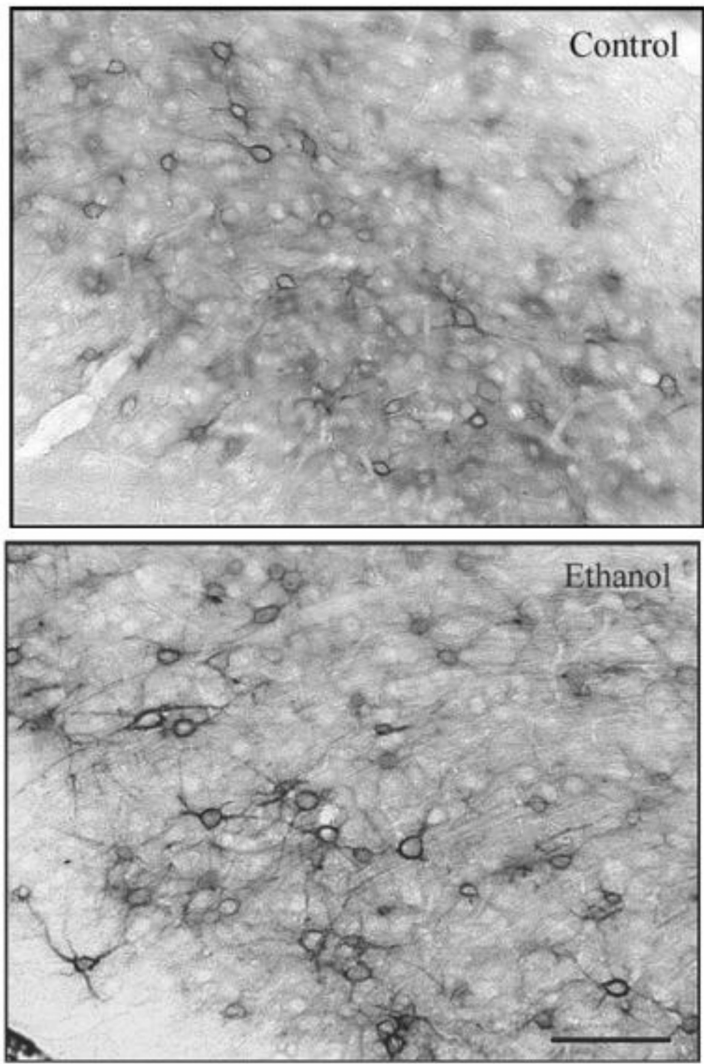

Figure 4.

\section{WFA Expession in Adult OFC}

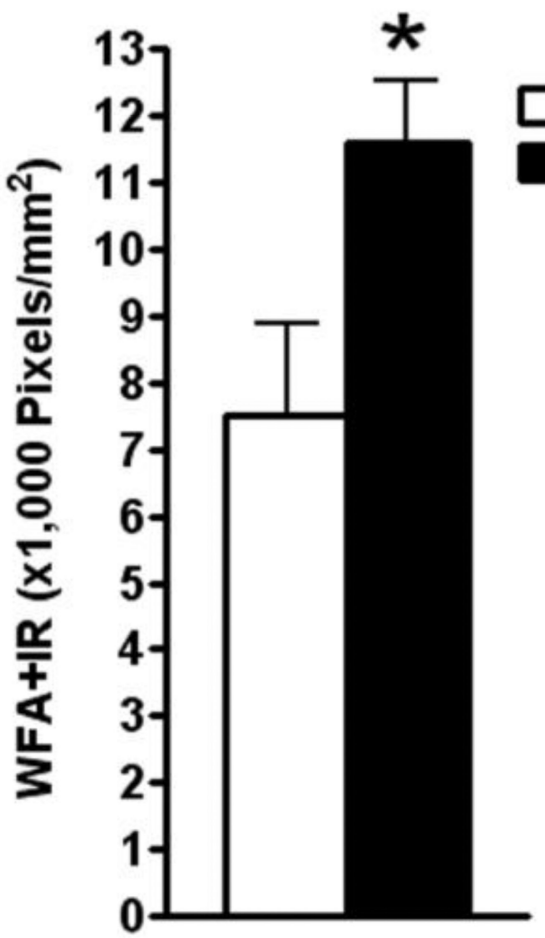

Adolescent intermittent ethanol (AIE) binge caused an increase in Wisteria floribunda agglutinin (WFA) immunoreactivity (IR) in the adult orbitofrontal cortex (OFC). Mice were given either water or ethanol $(5 \mathrm{~g} / \mathrm{kg}$, i.g.) in an intermittent fashion ( 2 days on -2 days off) during adolescence (P28-37). Immunohistochemistry was performed following sacrifice in adulthood (P110). (A) Representative images of WFA-IR in the OFC of control and AIE groups in adulthood show increased WFA-IR neural nets and increased intensity of staining in the AIE group. (B) Quantification of WFA-IR showed a significant increase in WFA-IR $*_{p}<0.05$, t-test. $\mathrm{N}=5$ Control, 5 AIE. Scale bare denotes 50 microns. 


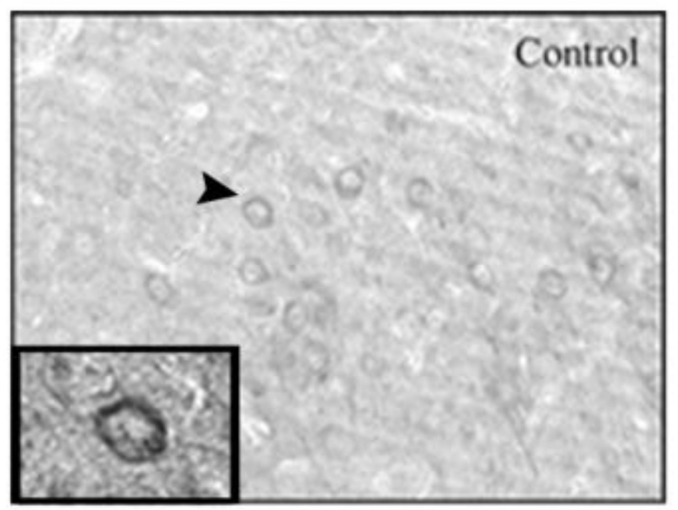

B
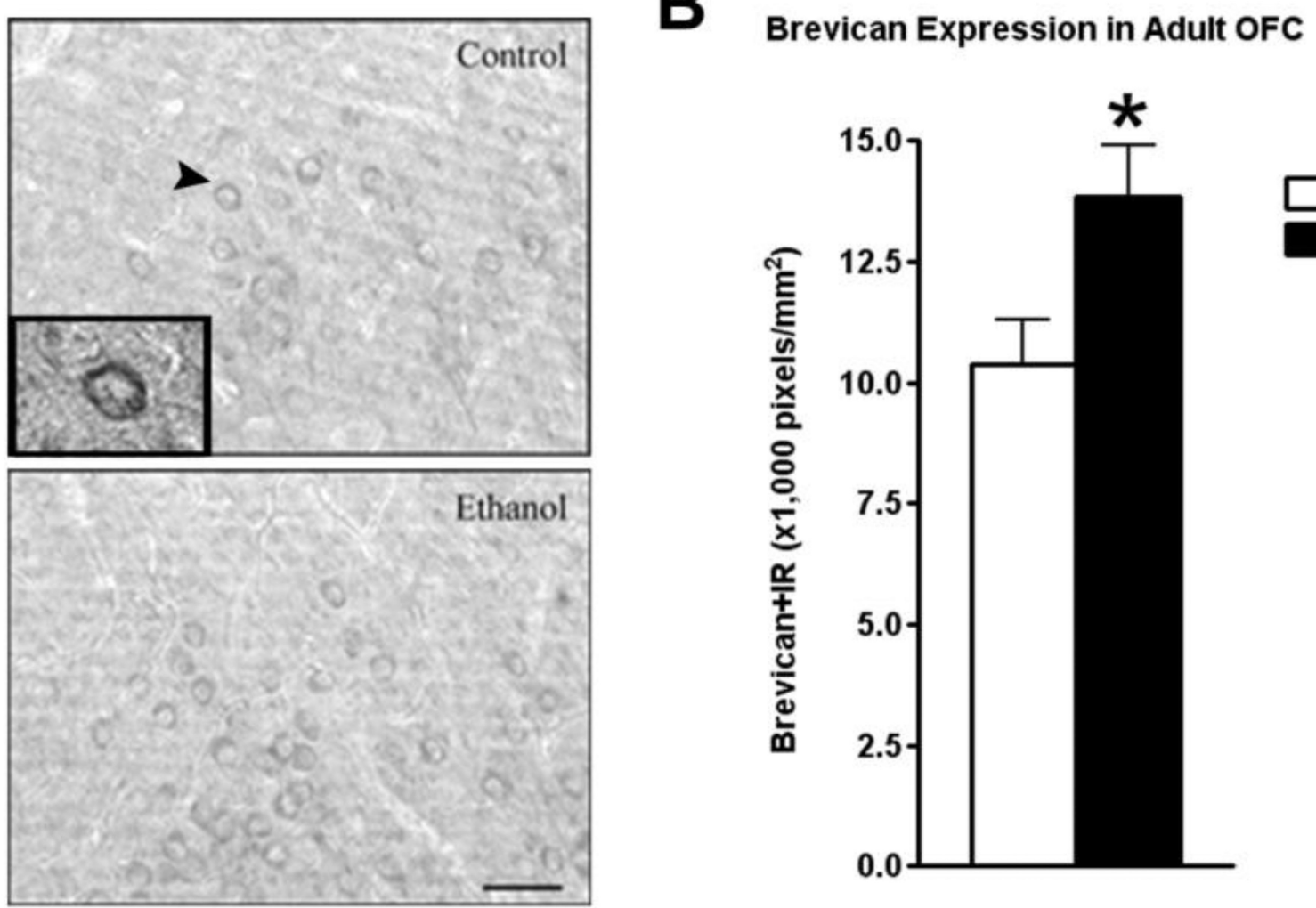

Figure 5.

Adolescent intermittent ethanol (AIE) binge caused an increase in brevican immunoreactivity (IR) in the adult orbitofrontal cortex (OFC). Mice were given either water or ethanol $(5 \mathrm{~g} / \mathrm{kg}$, i.g.) in an intermittent fashion (2 days on -2 days off) during adolescence (P28-37). Immunohistochemistry was performed following sacrifice in adulthood (P110).

(A) Representative images of brevican-IR in the OFC of control and AIE groups in adulthood show increased brevican-IR in the AIE group. A high magnification image of the cell surrounded by brevican identified by the arrowhead is included in the insert. (B) Quantification of brevican-IR showed a significant increase in brevican-IR $* p<0.05$, t-test. $\mathrm{N}=5$ Control, 5 AIE. Scale bare denotes 50 microns. 


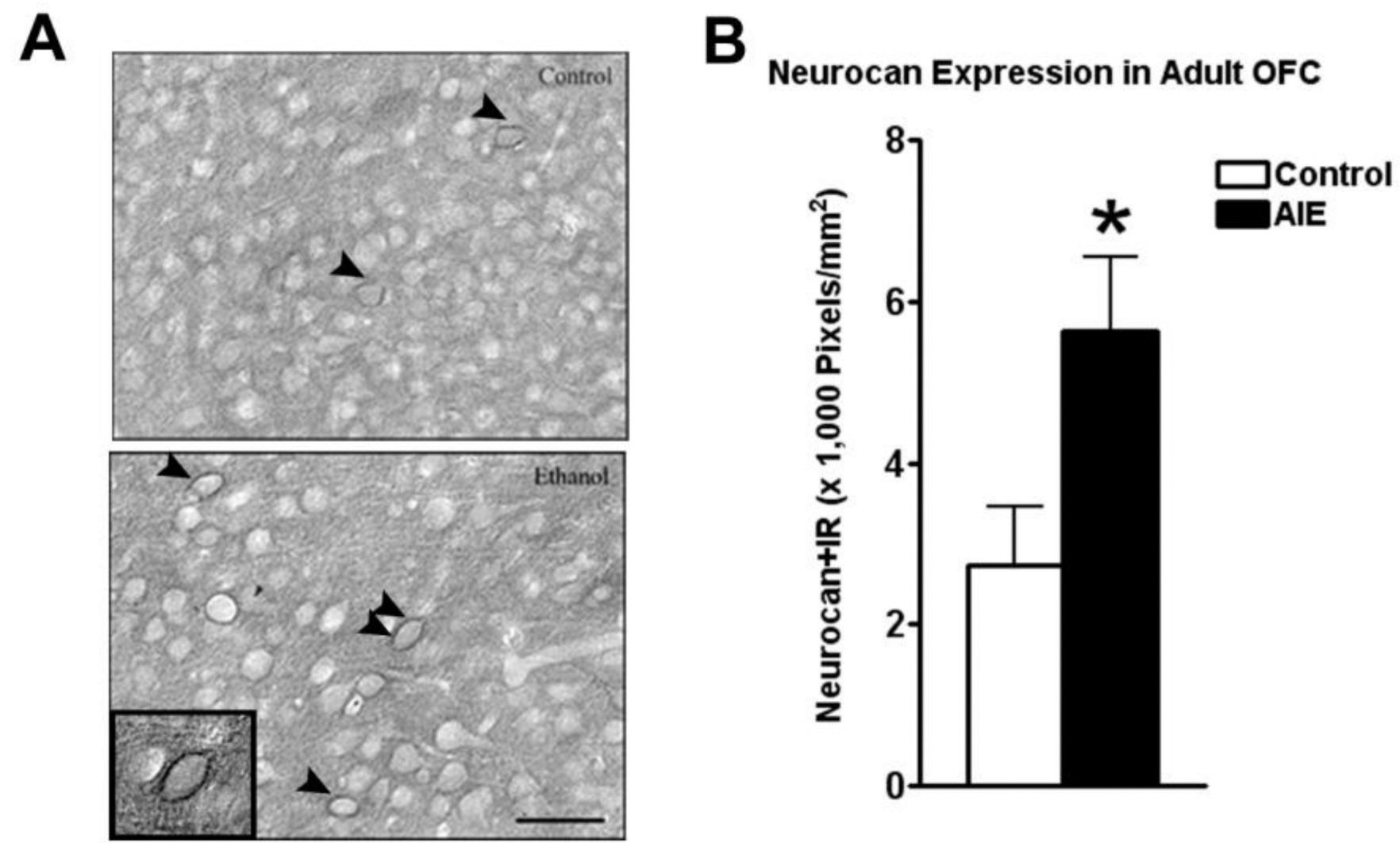

Figure 6.

Adolescent intermittent ethanol (AIE) binge caused an increase in neurocan immunoreactivity (IR) in the adult orbitofrontal cortex (OFC). Mice were given either water or ethanol $(5 \mathrm{~g} / \mathrm{kg}$, i.g.) in an intermittent fashion ( 2 days on -2 days off) during adolescence (P28-37). Immunohistochemistry was performed following sacrifice in adulthood (P110).

(A) Representative images of neurocan-IR in the OFC of control and AIE groups in adulthood show increased neurocan-IR in the AIE group. Arrowheads highlight cells surrounded by neurocan. A high magnification image of the cell identified by the double arrowhead is included in the insert. (B) Quantification of brevican-IR showed a significant increase in brevican-IR $* p<0.05$, t-test. $\mathrm{N}=5$ Control, 6 AIE. Scale bare denotes 50 microns. 
A

Expression of Phosphacan in Adult ofC

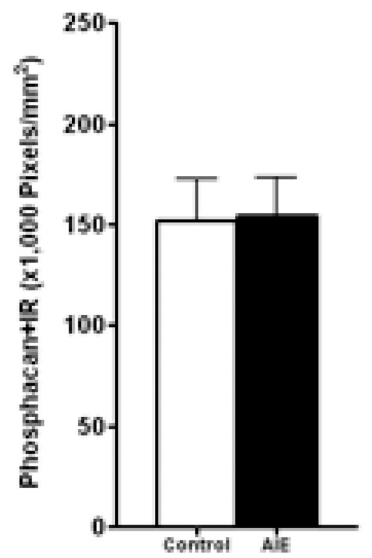

B

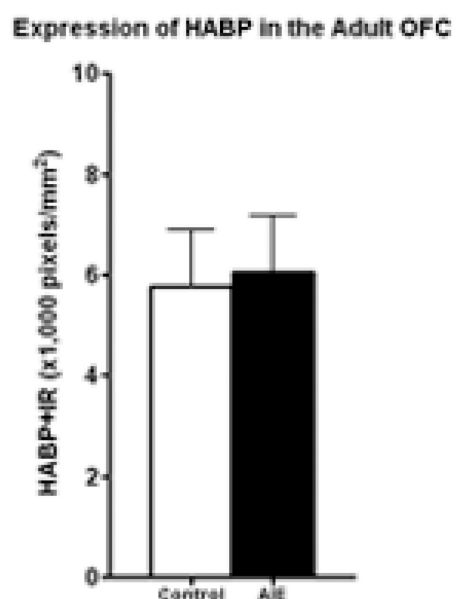

C

Expression of Tensacin-C in Adult OFC

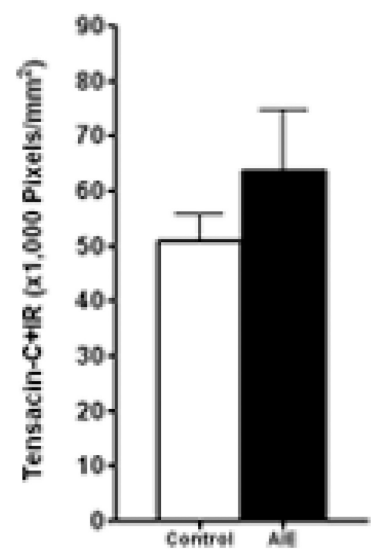

Figure 7.

Adolescent intermittent ethanol (AIE) binge did not cause any change in phosphocan, HABP, or tensacin-C immunoreactivity (IR) in the adult orbitofrontal cortex (OFC). Mice were given either water or ethanol $(5 \mathrm{~g} / \mathrm{kg}$, i.g.) in an intermittent fashion ( 2 days on -2 days off) during adolescence (P28-37). Immunohistochemistry was performed following sacrifice in adulthood (P110). (A) Quantification of phosphocan-IR showed no change between control and AIE groups $p=0.94$. (B) Quantification of HABP-IR showed no change between control and AIE groups $p=0.88$. (C) Quantification of Tensacin-C-IR showed no change between control and AIE groups $p=0.33$, t-test. $\mathrm{N}=5$ Control, 6 AIE. 


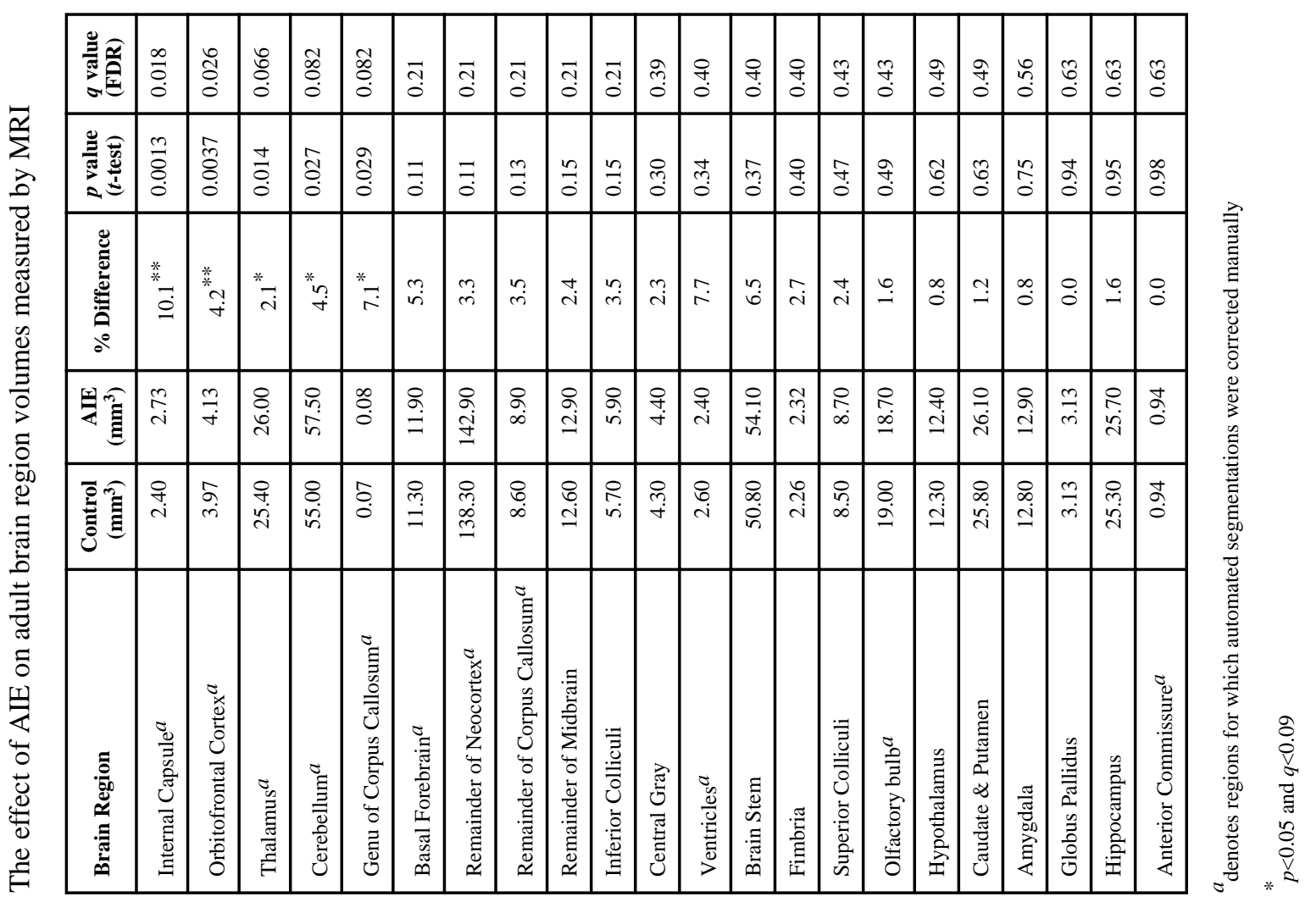

Pharmacol Biochem Behav. Author manuscript; available in PMC 2015 January 01. 


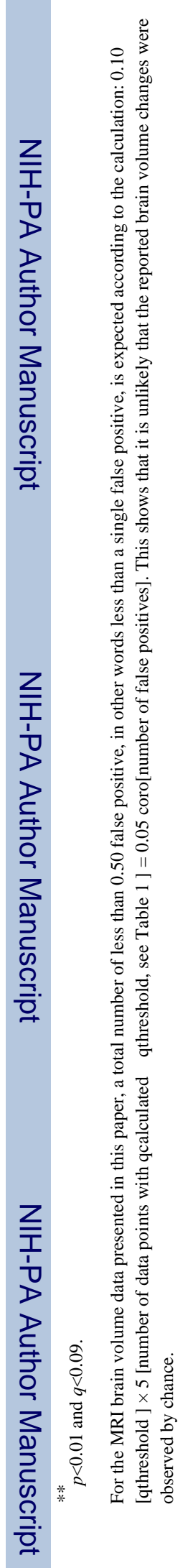

Pharmacol Biochem Behav. Author manuscript; available in PMC 2015 January 01. 


\section{Table 2}

The effect of AIE on the relative expression of extracellular matrix proteins (ECM) in the adult orbitofrontal cortex by IHC

\begin{tabular}{|c|c|}
\hline Extracellular Matrix Protein & \% Expression (AIE/Control) \\
WFA* $^{*}$ & $\mathbf{1 5 5}$ \\
Brevican $^{*}$ & $\mathbf{1 3 2}$ \\
Neurocan & \\
HABP & $\mathbf{2 0 5}$ \\
Phosphocan & 105 \\
Tenacin-C & 101 \\
\end{tabular}

Bold $p<0.05$, t-test, control to AIE. 